\title{
Laparoscopia e lavado peritoneal no pré-operatório do adenocarcinoma gástrico: podemos modificar a conduta?
}

\section{Preoperative laparoscopy and peritoneal lavage in gastric adenocarcinoma: can the approach be modified?}

Jorge Roberto Marcante Carlotto, TCBC-RS¹@; Fernanda Marcante Carlotto²@; Marcos Dal Vesco Neto3; Julia Pastorello4; lucas Duda Schmitz'; Juarez Antonio Dal-Vesco'

\section{R E S U M O}

\begin{abstract}
Objetivo: avaliar se a laparoscopia com lavado peritoneal é superior à tomografia computadorizada para o estadiamento do adenocarcinoma gástrico e se pode modificar a conduta cirúrgica do paciente. Métodos: estudo retrospectivo de 46 pacientes portadores de adenocarcinoma gástrico tratados pela equipe de cirurgia digestiva do Hospital de Clínicas de Passo Fundo (RS), de janeiro de 2015 a dezembro de 2018, e submetidos à laparoscopia com lavado peritoneal pré-operatório. Todos os pacientes foram submetidos ao estadiamento clínico pré-operatório com tomografia computadorizada. Resultados: dos 46 pacientes analisados, a maioria apresentava tumores localizados na cárdia $(34,8 \%)$, pouco diferenciados $(69,6 \%)$ e do subtipo células em anel de sinete $(65,2 \%)$. Em 91,3\% deles a tomografia computadorizada não identificou carcinomatose peritoneal ou metástases à distância. Entre estes pacientes com tomografia computadorizada negativa para doença à distância, 21,8\% apresentaram lavado peritoneal positivo para células neoplásicas e tiveram suas condutas terapêuticas modificadas. Conclusão: a laparoscopia e o lavado peritoneal alteraram a decisão cirúrgica em $21,8 \%$ dos pacientes, proporcionando um estadiamento pré-operatório mais fidedigno no adenocarcinoma gástrico.
\end{abstract}

Descritores: Adenocarcinoma. Neoplasias Gástricas. Laparoscopia. Lavagem Peritoneal. Período Pré-Operatório. Estadiamento de Neoplasias.

\section{INTRODUÇÃO}

C âncer gástrico tem sido descrito desde 3000 a.C., em inscrições hieroglíficas e manuscritos de papiros do Egito antigo. A primeira análise estatística importante de sua incidência e mortalidade, utilizando dados coletados em Verona, Itália (1760-1839), mostrou que o câncer gástrico era um dos mais comuns e letais à época'. Atualmente, cerca de 22.220 pacientes são diagnosticados anualmente nos Estados Unidos com esse tipo de neoplasia, dos quais 10.990 morrem em decorrência do tumor. Sua incidência, no entanto, é marcada por grande variabilidade geográfica, sendo que as áreas de alta incidência incluem a América Latina, Ásia Oriental e partes da Europa e do Oriente Médio². Em 95\% dos casos são adenocarcinomas e a maioria é diagnosticada como tumores avançados ${ }^{3}$.
Nos Estados Unidos e na Europa Ocidental, desde a década de 1970, a incidência de tumores distais vem diminuindo enquanto os da transição esofagogástrica e da cárdia vem aumentando ${ }^{4}$, estes com pior prognóstico e maior risco de disseminação peritoneal ${ }^{5}$.

Com o diagnóstico confirmado de adenocarcinoma, os pacientes devem ser estadiados para avaliação da extensão da doença e da conduta ${ }^{6}$. O estadiamento clínico pré-operatório inclui a tomografia computadorizada (TC) do abdome e, nos tumores localmente avançados, sugere-se complementar o estadiamento com laparoscopia e realização de citologia do lavado peritoneal $^{5}$. Além da TC, uma das técnicas complementares de diagnóstico que pode auxiliar na seleção dos doentes é a Tomografia Computadorizada com Emissão de Pósitrons (PET-TC).

1 - Hospital de Clínicas de Passo Fundo, Serviço de Cirurgia do Aparelho Digestivo, Passo Fundo, RS, Brasil. 2 - Universidade de Passo Fundo, Curso de Medicina, Passo Fundo, RS, Brasil. 3 - Hospital de Clínicas de Porto Alegre, Serviço de Cirurgia Geral, Porto Alegre, RS, Brasil. 4 - Hospital de Clínicas de Passo Fundo, Serviço de Oncologia Clínica, Passo Fundo, RS, Brasil. 
Entretanto, seu uso ainda não foi difundido em razão de que sua especificidade ainda não está bem definida e de que alguns tipos histológicos, como carcinomas mucinosos e de células em anel de sinete, apresentam baixa atividade metabólica 7 .

Estudos afirmam que 20\% a 30\% dos doentes com TC negativa apresentam metástases peritoneais à laparoscopia², confirmando que a TC é um método com baixa sensibilidade para detecção de metástases peritoniais ${ }^{7}$. Dessa forma, o objetivo da nossa pesquisa é avaliar se a laparoscopia com lavado peritoneal diagnóstico (LPD) é superior em relação à TC para o estadiamento do adenocarcinoma gástrico e se a mesma pode modificar a conduta cirúrgica do paciente.

\section{MÉTODOS}

Foram revisados retrospectivamente os prontuários de 46 pacientes com adenocarcinoma gástrico tratados por uma equipe de cirurgia digestiva do Hospital de Clínicas de Passo Fundo - RS, de janeiro de 2015 a dezembro de 2018. As variáveis estudadas foram demográficas, clínicas, endoscópicas, cirúrgicas e dos exames anatomopatológicos. Os dados foram coletados e organizados em planilhas de Excel. A análise estatística dos dados investigados foi realizada pelo SPSS Statistics 20.0 (IBM). As variáveis quantitativas foram apresentadas como média \pm desvio padrão e as variáveis qualitativas como frequência e porcentagem. A comparação entre os dados foi analisada pelo teste qui-quadrado de Pearson. Determinou-se o nível de significância em $5 \%(p=0,05)$.

O LPD era realizado com o paciente em posição de litotomia, sob anestesia geral, e pneumoperitônio com dióxido de carbono $\left(\mathrm{CO}_{2}\right)$ com pressão de $15 \mathrm{mmHg}$. O primeiro trocarte, de $10 \mathrm{~mm}$, era introduzido ao nível da cicatriz umbilical e dois trocartes de $5 \mathrm{~mm}$ eram colocados nos flancos direito e esquerdo.
A cavidade peritoneal era avaliada cuidadosamente à procura de metástases, o que incluía a investigação minunciosa do estômago, do omento, da superfície do fígado, de ambos os lados do diafragma, do mesentério e da bursa, através da abertura dos ligamentos gastrocólicos. Com a inspeção negativa para implantes macroscópicos, o lavado peritoneal era realizado em seguida, através da instilação de $350 \mathrm{ml}$ de soro fisiológico aquecido na retrocavidade dos epíplons e, após o vasculejo da cavidade peritoneal, o líquido era coletado e enviado para avaliação da citopatologia oncótica (Figura 1).

O trabalho foi aprovado previamente pelo Comitê de Ética da Instituição, tendo o parecer no 2186715 .

\section{RESULTADOS}

A maioria dos pacientes eram homens $(65,2 \%)$ com média de idade de 66,6 anos $( \pm 11,31)$ e o principal sintoma da neoplasia foi a epigastralgia (69,6\%). Conforme a classificação de Borrmann, a maioria das lesões era dos tipos II e III, localizadas principalmente no terço proximal, ao nível da cárdia (34,8\%) e com pesquisa de Helicobacter pylori negativa na maioria dos exames (91,3\%). Em relação à diferenciação histológica, houve predomínio dos tumores pouco diferenciados $(69,6 \%)$ e do subtipo células em anel de sinete $(65,2 \%)$. Constatou-se que $47,8 \%$ dos pacientes apresentavam linfonodomegalias na TC, principalmente perigástricas e em $91,3 \%$ deles, o exame de imagem não identificou metástases a distância.

Nos 46 pacientes que realizaram TC de abdome total para estadiamento de carcinoma de estômago e que realizaram LPD, houve concordância de resultado negativo entre a $\mathrm{TC}$ e a pesquisa de doença peritoneal macroscópica e microscópica em 32 pacientes $(69,6 \%)$, sendo considerados verdadeiro-negativos. 


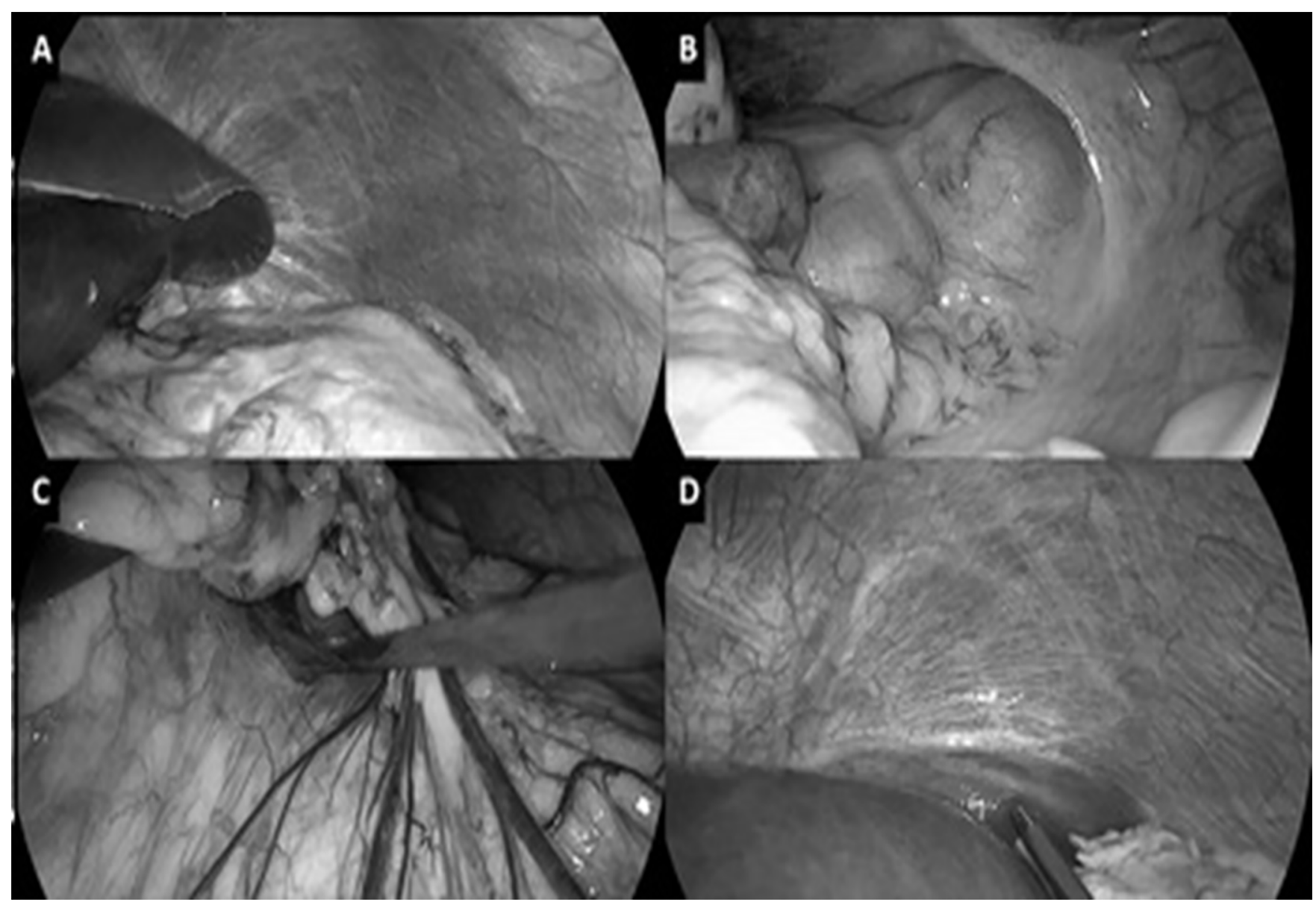

Figura 1. Laparoscopia diagnóstica com lavado peritoneal. A: visualização do peritônio visceral do estômago; B: avaliação do fundo de saco peritoneal; C: acesso à retrocavidade e realização da instilação do soro fisiológico; D: aspiração do soro instilado para análise citológica.

A concordância de resultados positivos entre os exames ocorreu em dois pacientes (4,3\%). Outros dois pacientes, que apresentavam TC positiva, tinham doença peritoneal negativa (4,3\%). E dez pacientes com TC negativa tinham LPD positivo para células neoplásicas, caracterizando a ocorrência de resultados falso-negativos nestes dez pacientes $(21,8 \%)$ (Tabela 1).
Quando analisamos o LPD, a diferenciação histológica estava relacionada com sua positividade $(p<0,05)$. Nos 32 pacientes com LPD e TC negativos para doença peritoneal e que foram submetidos à ressecção (69,5\%), a gastrectomia total foi a mais realizada, com uma média de ressecção de 35,8 linfonodos $( \pm 21,8)$. Os demais 14 pacientes, que foram identificados com TC ou doença peritoneal positivas, seguiram para tratamento quimioterápico $(30,5 \%)$.

Tabela 1. Análise dos pacientes que realizaram TC e seus respectivos resultados na citologia.

\begin{tabular}{lccc}
\hline & TC negativa $(\mathrm{n})$ & TC positiva $(\mathrm{n})$ & Total $(\mathrm{n})$ \\
\hline Doença peritoneal negativa $(\mathrm{n})$ & 32 & 2 & 34 \\
Doença peritoneal positiva $(\mathrm{n})$ & 10 & 2 & 12 \\
Total $(\mathrm{n})$ & 42 & 4 & 46 \\
\hline
\end{tabular}

TC: tomografia computadorizada. 


\section{DISCUSSÃO}

Juntamente com o desenvolvimento da cirurgia laparoscópica, o diagnóstico de laparoscopia com lavado peritoneal em cirurgia de câncer gástrico tem se tornado mais comum e sua viabilidade e sua utilidade têm sido, cada vez mais, relatadas em revisões sistemáticas. Devido à natureza menos invasiva dessa modalidade, o LPD pode ser realizado com poucas complicações, permitindo que os pacientes com doença peritoneal se recuperem mais rapidamente e recebam tratamento adequado o mais precocemente possível ${ }^{8}$.

A TC com contraste endovenoso é o método de imagem mais utilizado no estadiamento pré-operatório do câncer de estômago. No entanto, estudos prospectivos têm demonstrado que 20\% a $30 \%$ de pacientes considerados potencialmente curáveis, baseados em exames de imagem préoperatórios, apresentam doença metastática à cirurgia. Uma série de estudos recentes tem confirmado que a laparoscopia é extremamente sensível na detecção de metástases intra-abdominais em pacientes com câncer gástrico, evitando a realização de cirurgias desnecessárias em $21 \%$ a $41 \%$ dos pacientes ${ }^{9}$. A laparoscopia também permite a realização de lavado peritoneal e a obtenção de material para análise citológica, sendo que, a presença de células tumorais na cavidade peritoneal, mesmo na ausência de doença metastática macroscópica, é um fator de pior prognóstico ${ }^{10}$.

A sensibilidade da citologia do LPD para a detecção de doença peritoneal varia muito na literatura, de $26 \%$ a $70,8 \%$. Entretanto, essa variabilidade de resultados pode estar relacionada a métodos não padronizados de lavagem peritoneal, bem como, de vieses entre os observadores e entre as interpretações dos patologistas na análise da citologia ${ }^{11}$.
Pesquisas sugerem que o LPD pode ser rentável quando o procedimento é bem indicado. Considerando que a especificidade de metástases ocultas é próxima a 100\% e que a probabilidade de detecção de metástases ocultas pelo LPD após TC negativa é de $31 \%$, em consonância com outros trabalhos previamente publicados, verifica-se um aumento de quase duas vezes na probabilidade de custo-efetividade. Este resultado apoia o uso do procedimento quando os pacientes apresentam fatores de risco potenciais para doença oculta aos exames de imagem ${ }^{12}$.

Pesquisa realizada no Instituto do Câncer do Japão mostrou que o tratamento do câncer mudou para $47,4 \%$ dos pacientes submetidos ao LPD. Dessa forma, quando bem indicado, obteve um impacto muito maior na tomada de decisão clínica quando comparadas àquelas em estudos prospectivos anteriores. Com relação aos desfechos secundários, a acurácia diagnóstica do LPD atingiu $91,5 \%$ e a taxa de falso-negativo do procedimento foi de $10,6 \%{ }^{13}$.

A tomografia com emissão de pósitrons, não avaliada em nosso estudo, tem sido sugerida como modalidade de estadiamento apropriado para metástases à distância. Entretanto, a sensibilidade/ especificidade para detecção de metástase à distância foram relatadas como sendo de $21 \%$ e $40 \%$, respectivamente. Portanto, apesar destes exames, pacientes com câncer gástrico incurável ou irressecável ainda são submetidos à cirurgia não terapêutica. Dessa forma, para resolver esse problema, a laparoscopia diagnóstica tem sido defendida como essencial na tomada de decisões no câncer gástrico avançado. Lavados obtidos durante a laparoscopia diagnóstica foram usados como controles e a sua sensibilidade e especificidade chegou a $92 \%$ e $100 \%$, respectivamente ${ }^{14}$. 
As diretrizes do National Comprehensive Cancer Network (NCCN), de 2017, recomendam que a laparoscopia pré-operatória para detectar doença metastática oculta aos exames de imagem seja considerada em todos os pacientes com alto risco de metástases, ou seja, com tumores cT4, grandes tumores dos tipos III e IV, além dos tumores com metástase linfonodal2, uma vez que, a laparoscopia associada ao LPD na doença avançada, pode melhorar a tomada de decisão de tratamento para o câncer gástrico avançado e diminuir a cirurgia desnecessária ${ }^{14}$. Essas práticas são apoiadas por dados que sugerem que a doença M1 oculta é encontrada em até $40 \%$ dos pacientes com câncer gástrico e que a cirurgia não terapêutica pode ser evitada em um subconjunto significativo desses pacientes.
Uma coorte final do estudo incluiu apenas pacientes com câncer gástrico comprovado por biópsia que tiveram um estadiamento negativo inicial, laparoscopia seguida de quimioterapia ou quimiorradioterapia préoperatória e tentativa de gastrectomia. A laparoscopia diagnóstica de rotina no momento da ressecção definitiva em pacientes com câncer gástrico em tratamento pré-operatório pode prevenir a cirurgia não terapêutica em ao menos $12 \%$ de todos os pacientes e pode oferecer benefícios ainda maiores para pacientes de alto risco com tumores pouco diferenciados ${ }^{15}$.

Em nosso estudo, os achados da laparoscopia com LPD em nossos pacientes mudou a terapêutica inicial em dez (21,8\%) dos 46 pacientes e concluímos que deva ser realizada no estadiamento pré-operatório do adenocarcinoma de estômago.

\title{
A B S T R A C T
}

\begin{abstract}
Objective: to assess whether laparoscopy with peritoneal lavage is superior to computed tomography for staging gastric adenocarcinoma and whether it can modify the surgical approach. Methods: we conducted a retrospective study of 46 patients with gastric adenocarcinoma treated by the digestive surgery team of the Passo Fundo Clinics Hospital (RS), from January 2015 to December 2018, and submitted to laparoscopy with preoperative peritoneal lavage. All patients underwent preoperative clinical staging with computed tomography. Results: of the 46 patients analyzed, the majority had tumors located in the cardia (34.8\%), poorly differentiated (69.6\%), and subtype signet ring cells (65.2\%). In 91.3\%, the computed tomography scan did not identify peritoneal carcinomatosis or distant metastasis. Among these patients with negative computed tomography for distant disease, $21.8 \%$ had positive peritoneal lavage for neoplastic cells and had their therapeutic approaches modified. Conclusion: laparoscopy and peritoneal lavage altered the surgical decision in $21.8 \%$ of patients, providing a more reliable preoperative staging in gastric adenocarcinoma.
\end{abstract}

Keywords: Adenocarcinoma. Stomach Neoplasms. Laparoscopy. Peritoneal Lavage. Preoperative Period. Neoplasm Staging.

\section{REFERÊNCIAS}

1. Japanese Gastric Cancer Association. Japanese Classification of Gastric Carcinoma. 2nd English Edition. Gastric Cancer. 1998;1(1):10-24.

2. Ilhan E, Alemdar A, Ureyen O, Bas K. The importance of extensive intraoperative peritoneal lavage as a promising method in patients with gastric cancer showing positive peritoneal cytology without overt peritoneal metastasis and other therapeutic approaches. J Invest Surg. 2017;30(5):318-24.

3. Luinetti O, Fiocca R, Villani L, Alberizzi P, Ranzani GN, Solcia E. Genetic pattern, histological structure, and cellular phenotype in early and advanced gastric cancers: evidence for structure-related genetic subsets and for loss of glandular structure during progression of some tumors. Hum Pathol. 1998;29(7):702-9.
4. Montori G, Coccolini F, Ceresoli M, Catena F, Colaianni N, Poletti $E$, et al. The treatment of peritoneal carcinomatosis in advanced gastric cancer: state of the art. Int J Surg Oncol. 2014;2014:912418.

5. Debon LM, Viola LS, Toneto MG. Carcinomatose peritoneal em câncer gástrico: diagnóstico, manejo e conduta. Acta Méd. (Porto Alegre). 2018;39(2):82-91.

6. Hosogi $H$, Shinohara $H$, Tsunoda S, Hisamori S, Sumida $H$, Hida $K$, et al. Staging laparoscopy for advanced gastric cancer: significance of preoperative clinicopathological factors. Langenbecks Arch Surg. 2017;402(1):33-9.

7. Mosca AMSS, Barbosa LE. Abordagem terapêutica da carcinomatose peritoneal em doentes com carcinoma colorretal. Rev Port Cir. 2017;40:35-50. 
8. Irino $T$, Sano $T$, Hiki $N$, Ohashi $M$, Nunobe $S$, Kumagai $K$, et al. Diagnostic staging laparoscopy in gastric cancer: a prospective cohort at a cancer institute in Japan. Surg Endosc. 2018;32(1):268-75.

9. Koganti SB, Boddepalli S, Nambada M, Thumma VM, Nagari B, Sastry RA. Positive peritoneal lavage cytology - implications for staging and management of gastric cancer. Indian J Surg Oncol. 2016;7(4):430-5.

10. Frattini F, Rausei S, Chiappa C, Rovera F, Boni L, Dionigi $G$. Prognosis and treatment of patients with positive peritoneal cytology in advanced gastric cancer. World J Gastrointest Surg. 2013;5(5):135-7.

11. Machairas $N$, Charalampoudis $P$, Molmenti EP, Kykalos S, Tsaparas P, Stamopoulos P, et al. The value of staging laparoscopy in gastric cancer. Ann Gastroenterol. 2017;30(3):287-94.

12. Li K, Cannon JGD, Jiang SY, Sambare TD, Owens DK, Bendavid $E$, et al. Diagnostic staging laparoscopy in gastric cancer treatment: a cost-effectiveness analysis. J Surg Oncol. 2018;117(6):1288-96.
13. Yoon H, Lee DH. New approaches to gastric cancer staging: beyond endoscopic ultrasound, computed tomography and positron emission tomography. World J Gastroenterol. 2014;20(38):13783-90.

14. Ajani JA, D'Amico TA, Almhanna K, Bentrem DJ, Chao J, Ds P, et al. Gastric Cancer, Version 3.2016, NCCN Clinical Practice Guidelines in Oncology. J Natl Compr Canmc Netw. 2016;14(10):1286-312.

15. Thiels CA, Ikoma N, Fournier K, Das P, Blum M, Estrella JS, et al. Repeat staging laparoscopy for gastric cancer after preoperative therapy. J Surg Oncol. 2018;118(1):61-7.

Recebido em: 28/07/2019

Aceito para publicação em: 17/09/2019

Conflito de interesse: nenhum.

Fonte de financiamento: nenhuma.

\section{Endereço para correspondência:}

Fernanda Marcante Carlotto

E-mail: fmcarlotto@gmail.com

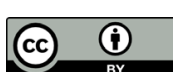

